\title{
270 ANTI-CD47 IMMUNOTHERAPY AS A THERAPEUTIC STRATEGY FOR THE TREATMENT OF BREAST CANCER BRAIN METASTASIS
}

${ }^{1}$ Jessica Mackert*, ${ }^{1}$ Elizabeth Stirling, ${ }^{1}$ Mitra Kooshki, ${ }^{2}$ Dawen Zhao, ${ }^{2}$ Alexandra Thomas, ${ }^{2}$ Glenn Lesser, ${ }^{2}$ Pierre Triozzi, ${ }^{1}$ David Soto-Pantoja. ${ }^{1}$ Wake Forest University, Winston-Salem, NC, USA; ${ }^{2}$ Wake Forest Baptist Health, Winston-Salem, NC, USA

Background Triple-negative breast cancer (TNBC) is a highly aggressive subtype of breast cancer characterized by a lack of specific targets and a 35\% incidence of brain metastasis. There is no targeted treatment for managing brain metastasis associated with TNBC; therefore, new strategies are urgently needed to overcome disease mortality. The presence of cell surface protein CD47 allows cancer cells to evade innate and adaptive immune surveillance resulting in metastatic spread. CD47 binds to and activates SIRP $\alpha$ on the surface of myeloid cells, inhibiting their phagocytic activity. On the other hand, CD47 binds the matricellular protein Thrombospondin-1 limiting $\mathrm{T}$ cell activation. Thus, blocking the CD47 is a potential therapeutic strategy for the prevention of brain metastasis.

Methods Breast cancer patient biopsies were stained with antibodies against CD47. An anti-CD47 antibody was used in a syngeneic model of orthotopic triple-negative breast cancer. CD47 null mice were used in a breast cancer brain metastasis model by intracardiac injection of the E0071-Br-Luc cell line. Brain metastatic burden was measured by the in vivo imaging system (IVIS) and quantified by luciferase luminescence. Immunohistochemical analysis was performed to quantify tumorinfiltrating macrophages. Gene expression analysis of tumors was carried out by RNA-sequencing.

Results Immunohistochemical staining of patient biopsies revealed an $89 \%$ increase in CD47 expression in metastatic brain tumors compared to primary lesions ( $p \leq 0.05$ ). AntiCD47 treatment in mice bearing brain metastatic 4T1br3 orthotopic tumors reduced tumor volume and tumor weight by over $50 \%$ compared to control mice $(\mathrm{p} \leq 0.05)$ and increased F4/80 macrophage marker 5-fold tumors compared to control ( $\mathrm{p} \leq 0.05)$. CD47 null mice had a $60 \%$ increase in survival $(\mathrm{p} \leq 0.05)$ and an $89 \%$ decrease in metastatic brain lesions ( $\mathrm{p} \leq 0.05$ ) compared to control mice in a brain metastasis model. Additionally, RNA sequencing revealed 318 uniquely expressed genes and a significant reduction of genes related to extracellular matrix organization in tumors treated with an anti-CD47 antibody.

Conclusions CD47 was increased in metastatic brain tumors compared to primary lesions in breast cancer patients. CD47 blockade reduced orthotopic brain-metastatic tumor burden associated with increased macrophage infiltration and reduced extracellular matrix-associated gene expression. Additionally, CD47 null mice had improved overall survival and reduced brain metastatic lesions. Thus, CD47 blockade may be an effective therapeutic for triple-negative breast cancer brain metastasis.

Acknowledgements This work is supported by a NCI R21 CA249349 and the American Cancer Society Research Scholar Grant (133727-RSG-19-150-01-LIB).

Ethics Approval Animal studies were approved by the Wake Forest School of Medicine Animal Care and UseCommittee (ACAUC). Human subject studies were approved by the institutional review board (IRB).

http://dx.doi.org/10.1136/jitc-2021-SITC2021.270 